\title{
Photonic Liquid Crystal Fibers with Polymers
}

T.R. Woliński ${ }^{a}$, M. Tefelska $^{a}$, K. Mileńko $^{a}$, A. Siarkowska ${ }^{a, *}$, D. Budaszewski ${ }^{a}$, A.W. Domański ${ }^{a}$, S. ERTMAN $^{a}$, K. Orzechowski ${ }^{a}$, K. RutKOWska $^{a}$, M. Sierakowski $^{a}$, E. Nowinowski-Kruszelnicki ${ }^{b}$, R. DĄBrowski ${ }^{b}$ And P. Mergo ${ }^{c}$

${ }^{a}$ Faculty of Physics, Warsaw University of Technology, Warsaw, Poland

${ }^{b}$ Military University of Technology, Warsaw, Poland

${ }^{c}$ Maria Curie Skłodowska University, Lublin, Poland

Photonic liquid crystal fibers with polymers constitute a new solution based on liquid crystals and microstructured polymer optical fibers opening up new areas in innovative sensing and photonic devices applications. Compared with their silica-based microstructured fibers, it is easier to fabricate exotic microstructured polymer optical fibers by extrusion or drilling at low temperature; their nonlinearity is potentially stronger, the range of available polymers that may be drawn is more diverse and the biocompatibility of polymers is often better. Liquid crystals due to their attractive properties i.e., the high birefringence, high electro-optic and thermo-optic effects are a very good candidate for microstructured polymer optical fiber infiltration to obtain tunable all-in-fiber innovative photonic devices. The paper will discuss basic properties and possible applications of the polymer photonic liquid crystal fibers that will arise from their high optical tunability with external and internal factors. Current research effort is directed towards two main solutions: photonic crystal fibers and microstructured polymer optical fiber-based structures, both infiltrated with liquid crystals of tailored optical properties.

DOI: 10.12693/APhysPolA.124.613

PACS: 42.70.Df, 42.81.Wg, 02.60.Cb, 42.70.Qs

\section{Introduction}

Photonic liquid crystal fibers (PLCFs) in recent years have attracted growing interest over the world due to its properties. PLCF merge structure of photonic crystal fibers with electrical, thermal and optical properties of liquid crystals (LCs). This combination allowed to obtain new applications [1-4]. Mostly to produce PLCFs there has been used silica glass, where preform is prepared by capillary stacking or casting using sol-gel techniques. Recently other materials to produce fibers are being used i.e. polymers, which lead to creating polymer optical fibers (POFs). In contrast to silica-glass optical fiber, polymer fiber has very large diameter, where cores size can be almost $96 \%$ of the cross-section. Such large core, that can be even 100 time larger than in silica fiber, allows easy alignment as well as easier connecting to other fibers. Despite all these benefits POF has not been used worldwide in telecommunication network. The main reason for this is very high modal dispersion that comes with large core. Construction of single mode POF, which could eliminate this problem, provided to be hard, because of small mode area limits. Also there exists problem with choosing proper polymer for fabrication. Mostly used are fluorinated polymer, because of their absorption losses in material. However, even with this kind of polymers, the use of POF is limited to few hundred meters. The next step in fiber development was creating microstructured polymer optical fibers (mPOFs) [5]. Unlike the traditional POFs, mPOFs guiding mechanism rather than arises from variations in refractive index of material, has pattern of microscopic air channels

*corresponding author; e-mail: siarkowska@if.pw.edu.pl that goes along the full length of the fiber. As may be noted, this is similar mechanism that can be observed in photonic crystal fibers. Compared with their silica-based microstructured predecessors, mPOFs are easier to fabricate by extrusion or drilling at low temperature.

This paper will discuss basic properties and possible applications of the polymer photonic liquid crystal fibers that will arise from their high optical tunability with external and internal factors. Current research effort is focused on researching electrical and thermal tuning of photonic crystal fibers and mPOF-based structures, both infiltrated with liquid crystals of tailored optical properties.

First microstructured polymer based fiber was made of PMMA by Eijkelenborg et al. [6]. Since then POFs were produced from various types of polymers i.e. PC, Topas and biodegradable polymers. Those kind of polymers are called optical polymers. They are mostly used for their low cost, high impact resistance and ability to integrate optical and mechanical features [7]. Below we present three most frequently used materials. Comparison of their refractive indexes is shown in Fig. 1.

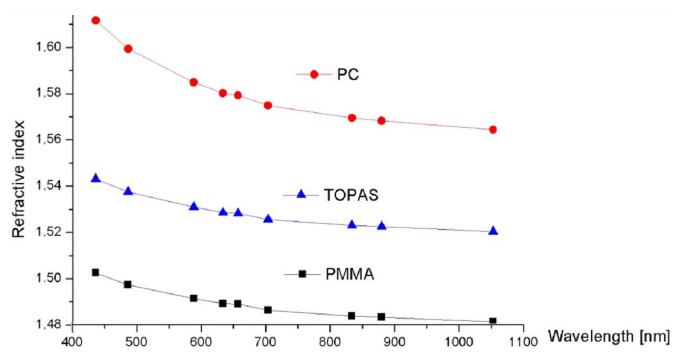

Fig. 1. Refractive indexes of polymers: PMMA, PC, and Topas (data from [7]).

\section{1. $P M M A$}


Polymethyl methacrylate (PMMA) is most commonly used to create mPOFs, mainly because of its optical properties. PMMA has refractive index of 1.4914 at $587.5 \mathrm{~nm}$ and it shows high transparency (about 93\% for a thickness of $3 \mathrm{~mm}$ ) in visible range. It has high mechanical straight, but on the other hand resistance to shock is relatively low and after critical stress is exceeded, polymer starts to crumble. Also it has low operating temperature (below $80^{\circ} \mathrm{C}$ ), which limits its applications. Nevertheless material properties of PMMA provide advantages in comparison with silica in the fabrication of fibers. It is lighter and more durable than silica fibers with high flexibility - it can be strained more than $30 \%$ without breaking [8].

\section{2. $P C$}

Polycarbonate (PC) is, next to the PMMA, most commonly used polymer to create mPOFs. PC has refractive index of 1.5849 at $588 \mathrm{~nm}$ and light transmittance of $87 \%$ (for a thickness of $3 \mathrm{~mm}$ ) for visible range. The characteristics of PC are similar to those of PMMA, but polycarbonite is stronger and more stable in greater temperature range, up to $155^{\circ} \mathrm{C}$. Contrary to most thermoplastics, PC can undergo plastic deformations without breaking. In result it can be processed in room temperature, which can be used for fabrication transparent parts, that cannot be made of sheet metal.

\subsection{Topas}

Topas is the trade name of family of cyclic olefin copolymers (COCs) from TOPAS Advanced Polymers. COCs are made from ethylene and norbornene, which makes it an amorphous copolymer with a cyclic olefin structure. They also have many advantages compared to previously described polymers. Topas has greater heat resistance than $\mathrm{PC}$ and it has very high moisture barrier which, with low water absorption, allows to avoid problem during drawing PMMA due to bubble formation. Topas has high transparency in the visible and near ultraviolet regions coupled with refractive index of 1.53 . This parameter makes it attractive for optical applications like components for cameras. Furthermore this polymer has low birefringence, is has good chemical resistance to common solvents like acetone and low stress-optical constant in the same range as PMMA. More information about Topas can be found in [9-12].

\section{Polymer photonic crystal fiber}

The use of polymers to create microstructured fibers allowed a wide variety of fibers structures to be constructed, which in result made it necessarily to create new methods of fabricating them. Many materials can be used to create mPOFs preforms. The best-known technique to fabricate $\mathrm{mPOFs}$ is similar to silica glass PCF manufacturing technology, although in case of polymer materials this process requires lower temperatures. Fabrication process of mPOF consist of two steps. First one is creating several centimeter width preform and the second is extrusion of previously created preform. The lower end of preform is heated to $200^{\circ} \mathrm{C}$ and its diameter is reduced to final diameter of created fiber. In the end the polymer coating is applied to fiber. The extrusion process can be continuous or batch. The former process requires that a monomer, an initiator and chain transfer agent are continuously put into the reactor and fiber is continuously withdrawn from the die. The process can be different in details based on the type of manufactured fiber [13]. Different techniques to create mPOFs, other than extrusion, are: the interfacial gel polymerization technique [14], the diffusion technique [15], drilling [16], and injection moulding [17]. Fabrication processes of mPOF have the advantage over glass fiber manufacturing. The main reason for that is, apart from lower processing temperature and cheaper materials, polymer fibers are not restricted by close packed arrangement of circular holes, which in result hole structure is not restricted to hexagonal or square package (as in PCF) and allows to create different material modification.

Apart from fabrication advantages compared to silica fibers, mPOFs have also superiority in other fiber properties. While in glass fibers possibilities for modifying the properties by doping are limited by high processing temperature, polymers can be specifically designed. Also mPOFs can be fabricated from single polymer without the use of dopants and as a result wider choice of polymers are available [18]. Example of other properties are: stronger nonlinearity and better biocompatibility. These features can be used in fiber optics devices and sensors.

Next step will be to infiltrate holes of mPOF with LC to create polymer liquid crystal fiber (Pol-PLCF). However in this new kind of fiber acquiring desired molecules orientation is problematic, because most organic solvents and often high temperature, may destruct delicate structure of polymer fiber $[19,20]$.

\section{Experimental results}

All mPOF's used in our experiments were manufactured at Kiriama company. Those are FM-340 and SM-340. Their cladding is made of PMMA and outer coating is made of PC. Core structure is composed of 6 concentric rings of air-holes. Other parameters are shown in Table.

\section{TABLE}

Comparison of parameters of polymer photonic crystal fibers FM-340 and SM-340.

\begin{tabular}{c|c|c}
\hline \hline Parameter & FM-340 & SM-340 \\
\hline diameter $[\mu \mathrm{m}]$ & 340 & 340 \\
core diameter $[\mu \mathrm{m}]$ & 15 & 8 \\
loss $650 \mathrm{~nm}[\mathrm{~dB} / \mathrm{m}]$ & 1.0 & 1.6
\end{tabular}

Both fibers were infiltrated with $1781 \mathrm{~A}-2$ ' LC mixture fabricated at the Military University of Technology in Warsaw. It has modified chemical composition made to prevent interacting with PMMA polymer. One of the advantages of this $\mathrm{LC}$ is that in wide range of temperatures its ordinary refractive index is close to refractive index of the PMMA (Fig. 2). However at this moment controlling orientation is not possible. We can assume that 


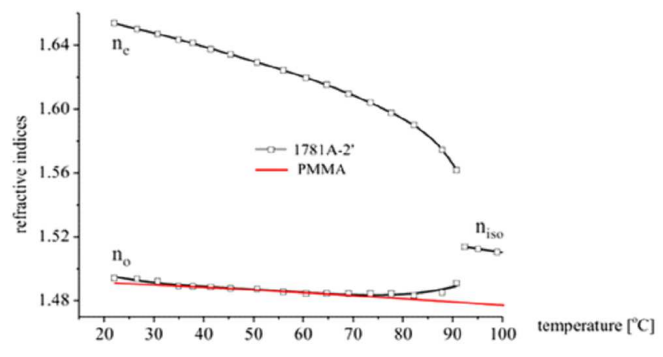

Fig. 2. Refractive index of $1781 \mathrm{~A}-2^{\prime}$ LC in function of temperature.

orientation is planar, because the fiber was infiltrated by flow. This conclusion is confirmed by the following experiments.

The length of the infiltrated part in both fibers was about $30 \mathrm{~mm}$ for FM-340 and about $3 \mathrm{~mm}$ for SM-340. In experiment one section of fibers were heated with Peltier module and results were collected by spectrometer Ocean Optics USB4000 with an $0.2 \mathrm{~nm}$ optical resolution. As light source halogen lamp (Ocean Optics Mikropack) for FM-340 and supercontinuum for SM-340 was used (Fig. 3).

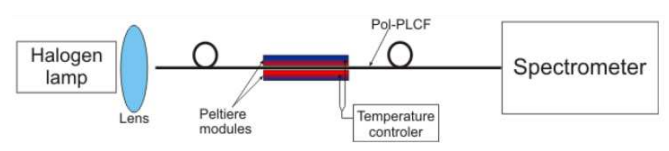

Fig. 3. Experimental setup for measuring temperature tuning.

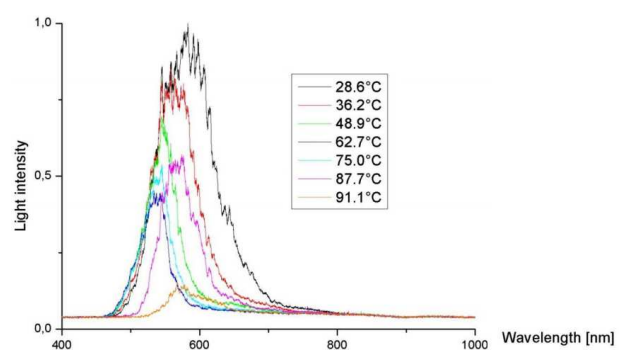

Fig. 4. Temperature tuning of photonic band gaps within the temperature range from $28.6^{\circ} \mathrm{C}$ to $91.1^{\circ} \mathrm{C}$ for FM-340.

After heating the temperature tuning of photonic band gaps for the range $28.6^{\circ} \mathrm{C}$ to $91.1^{\circ} \mathrm{C}$ (for $\mathrm{FM}-340$ ) and from $27^{\circ} \mathrm{C}$ to $92^{\circ} \mathrm{C}$ (for SM-340) can be observed. Figure 4 shows results for FM-340. It can be seen that with increasing temperature the band gap is shifting toward lower wavelengths. The maximum shift is obtained for $\approx 62{ }^{\circ} \mathrm{C}$. Also the narrowing of photonic band gap can be noticed. The maximum of obtained band gap occurs for $582.79 \mathrm{~nm}$ wavelength. Increase of temperature results in changing the band gap. Afterwards for temperature $87.7^{\circ} \mathrm{C}$ the new band gap appears with maximum for $573.99 \mathrm{~nm}$ wavelength. The change of wavelength after is about $0.15 \mathrm{~nm} /{ }^{\circ} \mathrm{C}$. On the other hand, for SM-340

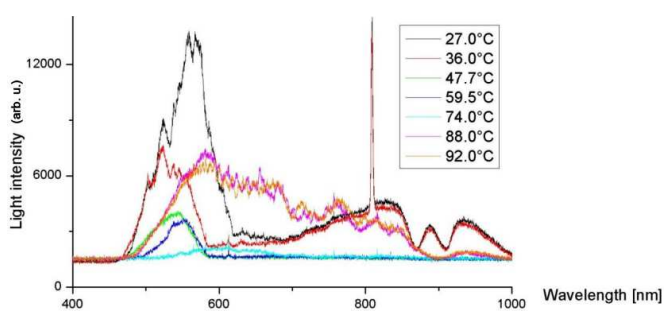

Fig. 5. Temperature tuning of photonic band gaps within the temperature range from $27^{\circ} \mathrm{C}$ to $92{ }^{\circ} \mathrm{C}$ for SM-340.

(Fig. 5) we can observe slightly different results. The first part up to $700 \mathrm{~nm}$ represents propagation in core and the second part behind $700 \mathrm{~nm}$ propagation in cladding. Single peak is so called "spike from pump". We can see that for single mode fiber attenuation is higher than in multimode fiber and here maximum shift is obtained for $\approx 36^{\circ} \mathrm{C}$. Here the maximum of obtained band gap occurs for $558.79 \mathrm{~nm}$ wavelength. After increase of temperature to $87.7^{\circ} \mathrm{C}$ the new band gap appears with maximum for $580.95 \mathrm{~nm}$ wavelength. The change of wavelength after is about $0.36 \mathrm{~nm} /{ }^{\circ} \mathrm{C}$.

Second experiment involved electrical tuning of the FM-340 fiber. Here mPOF was infiltrated with 5CB LC. The experimental setup is similar to one shown in Fig. 3. Only difference is that instead of the Peltier modules and temperature controller it consists of capacitor plates, amplifier and generator producing sinusoidal signal of frequency $500 \mathrm{~Hz}$.

Figure 6 shows electrical tuning of photonic band gaps. We can observe that molecules reorientation occurs. We can assume that orientation is planar, because the fiber was infiltrated by flow. Other than that we can also observe that for higher fields values band gap narrowed and changed its maximum length. Still only one band gap can be observed. The maximum for obtained band gap occurs for $750 \mathrm{~nm}$ wavelength. During the reorientation process the band gap vanishes. Afterwards for electric field of value $3.44 \mathrm{~V} / \mu \mathrm{m}$ the new band gap appears. Its maximum is for $696 \mathrm{~nm}$ wavelength and it has much lower light intensity. The change of wavelength after molecules reorientation is about $16 \mathrm{~nm} / \mathrm{V}$. In silica glass filled with $5 \mathrm{CB}$ many more band gaps can be seen. It is possible that in case of polymer material there are more band gaps, but they are attenuated. In here high voltage was used to obtain results. The reason for this was long distance between capacitor plates and photonic structure. The solution could be to deluge electrodes into the fiber.

Additionally, Fig. 7 shows comparison of light spectrum in empty and filled with LC fiber. We can observe that presence of $\mathrm{LC}$ reduces the intensity of light. The light color lines at the bottom represent propagation in the cladding.

Furthermore, the first attempts on mPOF fibers created at University of Marie Curie-Skłodowska in Lublin, Poland, have been undertaken. Fibers were made entirely from PC. The light was not put directly, but through 


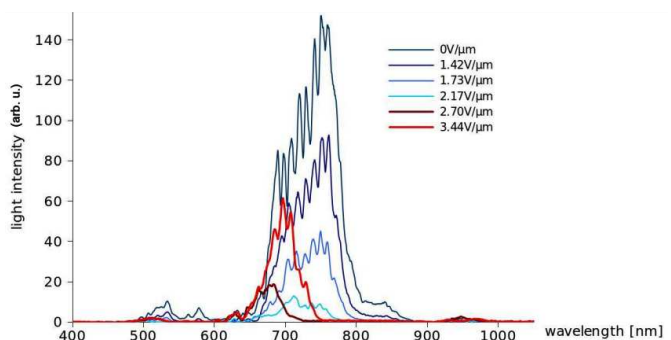

Fig. 6. Electrical tuning of photonic band gaps for FM-340.

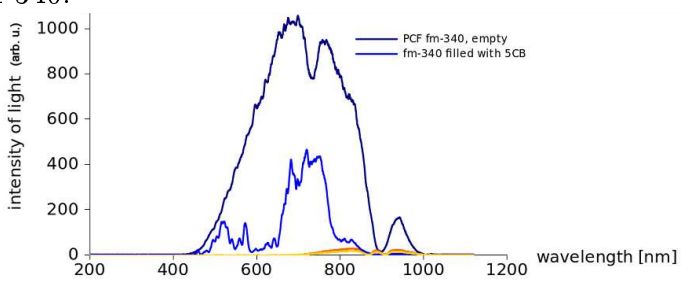

Fig. 7. Light spectrum for empty and filled with $5 \mathrm{CB}$ LC photonic crystal fiber FM-340.

coupling with single mode glass fiber SM600. This was necessary because in other case the propagation could not be achieved. The light source was $633 \mathrm{~nm}$ laser. Figure 8 shows very weak core propagation. So far it has not been possible to obtain localized light propagation in the core. Further experiments are in progress.

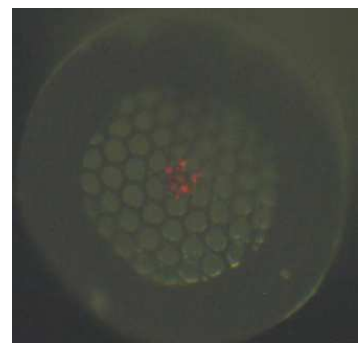

Fig. 8. Light propagation in mPOF created at University of Marie Curie-Skłodowska in Lublin, Poland.

\section{Conclusions}

Electrical and thermal tuning of Pol-PLCFs have been demonstrated. For the fiber with larger core (FM-340) it can be seen that an increasing temperature shifts the photonic band gap towards lower wavelengths. In case of the single mode fiber (SM-340) attenuation is much higher than in the multimode fiber and the maximum shift is reduced to half of the value for FM-340. Due to small core dimensions the infiltration process was relatively long in comparison to large-core fibers. Electric field induced molecules reorientation required high voltage that could be reduced by introducing electrodes directly into the fiber microstructure.

The use of polymer photonic crystal fibers creates an opportunity for a wide variety of flexible fiber structures that can find telecommunication, sensors, and optical devices applications. Liquid crystal introduced into the microstructured polymer fiber creates even more possibilities: photonic band gap tuning demonstrated in this paper could be used to fabricate e.g. fiber optic tunable filters.

\section{Acknowledgments}

This work is supported by the Polish National Center of Science under the grant no. 2011/01/B/ST7/05015.

\section{References}

[1] T.R. Woliński, S. Ertman, D. Budaszewski, M. Chychłowski, A. Czapla, R. Dąbrowski, A.W. Domański, P. Mergo, E. Nowinowski-Kruszelnicki, K.A. Rutkowska, M. Sierakowski, M. Tefelska, Photon. Lett. Poland 3, 20 (2011).

[2] S. Ertman, A.H. Rodríguez, M.M. Tefelska, M.S. Chychłowski, D. Pysz, R. Buczyński, E. NowinowskiKruszelnicki, R. Dąbrowski, T.R. Woliński, IEEE 30, 1208 (2011).

[3] M.M. Tefelska, M.S. Chychłowski, T.R. Woliński, R. Dąbrowski, W. Rejmer, J. Wójcik, Acta Phys. Pol. A 118, 1259 (2010).

[4] A. Siarkowska, S. Ertman, T.R. Woliński, Proc. SPIE 8454, 845411 (2011).

[5] A. Argyros, J. Lightwave Technol. 27, 1571 (2009).

[6] M.A. Eijkelenborg, M.C.J. Large, A. Argyros, J. Zagari, S. Manos, N.A. Issa, I. Bassett, S. Fleming, R.C. McPhedran, C.M. de Sterke, N.A.P. Nicorovici, Opt. Expr. 9, 319 (2001).

[7] N.G. Sultanova, S.N. Kasarova, I.D. Nikolov, Opt. Quant. Electron. 45, 221 (2013).

[8] A. Filipkowski, D. Pysz, P. Gdula, K. Welikow, K. Harasny, A.J. Waddie, K. Borzycki, A. Kraft, R. Piramidowicz, R. Stepien, M.R. Taghizadehb, R. Buczynski, Proc. SPIE 8426, 842616 (2012).

[9] G. Emiliyanov, J.B. Jensen, O. Bang, P.E. Hoiby, L.H. Pedersen, E.M. Kjaer, L. Lindvold, Opt. Lett. 32, 460 (2007).

[10] A. Stefani, K. Nielsen, H.K. Rasmussen, O. Bang, Opt. Commun. 285, 1825 (2012).

[11] C. Markos, A. Stefani, K. Nielsen, H.K. Rasmussen, W. Yuan, O. Bang, Opt. Expr. 21, 4758 (2013).

[12] G. Khanarian, H. Celanese, Opt. Eng. 40, 1024 (2001).

[13] M.A. Eijkelenborg, A. Argyros, G. Barton, I.M. Bassett, M. Fellew, G. Henry, N.A. Issa, M.C.J. Large, S. Manos, W. Padden, L. Poladian, J. Zagari, Opt. Fiber Technol. 9, 199 (2003).

[14] J. Zubia, J. Arrue, Opt. Fiber Technol. 7, 101 (2001).

[15] R. Nakao, A. Kondo, Y. Koike, J. Lightwave Technol. 30, 969 (2012).

[16] G. Barton, M.A. Eijkelenborg, G. Henry, M.C.J. Large, J. Zagari, Opt. Fiber Technol. 10, 325 (2004).

[17] Y. Zhang, K. Li, L. Wang, L. Ren, W. Zhao, R. Miao, Opt. Expr. 14, 5541 (2006).

[18] M.C.J. Large, D. Blacket, C.A. Bunge, IEEE Sensors J. 10, 1213 (2010).

[19] W. Yuan, L. Wei, T.T. Alkeskjold, A. Bjarklev, O. Bang, Opt. Expr. 17, 19356 (2009).

[20] Y. Zhang, L. He, H. Ji, S. Yang, M. Chen, S. Xie, Optoelectron. Lett. 3, 47 (2007). 